\title{
Diseño de iluminación: desarrollo, práctica y educación
}

Víctor Palacio

\section{Resumen}

El diseño de lluminación es una profesión de reciente creación que congrega a diversos participantes, todos ellos con un alto interés por la luz y sus aplicaciones, así como especialistas que ponen en práctica un conjunto de conocimientos sistematizado para iluminar el espacio arquitectónico y educadores con capacidad auto formativa y vocación para transmitir lo aprendido de forma teórica y experimental.

En este artículo se comparten datos sobre los orígenes del diseño de iluminación, su evolución y constitución como una profesión formal.

Palabras clave: luz, iluminación, diseño, espacio, arquitectura, Richard Kelly.

\section{LIGHTING DESIGN: DEVELOPMENT, PRACTICE AND EDUCATION}

\begin{abstract}
Lighting design is a newly created profession that gathers diverse participants, all of them with a high interest in light and its applications, as well as specialists who put into practice a set of systematized knowledge to illuminate the architectural space and educators with the ability to formative self and vocation to transmit what has been learned in a theoretical and experimental way.

In this article we share data about the origins of lighting design, its evolution and constitution as a formal profession.
\end{abstract}

Key words: light, lighting, design, space, architecture, Richard Kelly. 
Diseñador de iluminación arquitectónica, dirige la firma "ideas en luz"; fue presidente de la International Association of Lighting Designers (IALD) en los años 2016 y 2017; cuenta con la certificación Certified Lighting Designer (CLD). Su filosofía de diseño consiste en colaborar en la creación de ambientes luminosos únicos en beneficio de las personas.

Su experiencia de más de 20 años en proyectos de iluminación incluye museos, espacios comerciales, proyectos urbanos, residenciales, educativos y corporativos. Ha sido profesor en cursos de museografía del Instituto Nacional de Antropología e Historia, del Instituto Nacional de Bellas Artes y de la Maestría de Museos de la Universidad Iberoamericana; y ha coordinado los diplomados Espacio e lluminación y Dilux. Conferencista en los seminarios de la llluminating Engineering Society (IES) de México, Lightfair, PLDC, Enlighten Americas y el Encuentro Iberoamericando de Lighting Design.

https://orcid.org/0000-0001-6300-6950

\section{Comencemos con algunas definiciones...}

\section{Luz}

Técnicamente se define como la banda del espectro electromagnético que es visible por el ojo humano, es decir, se trata de energía radiante que tiene la capacidad de estimular nuestra visión. Más allá de esta definición, la luz tiene diversos sentidos tanto en el mundo físico como en el intelectual, emocional y espiritual. La luz representa el conocimiento, la verdad, la claridad, la presencia de lo divino y una guía en la oscuridad. Todas estas ideas, desarrolladas por el ser humano e

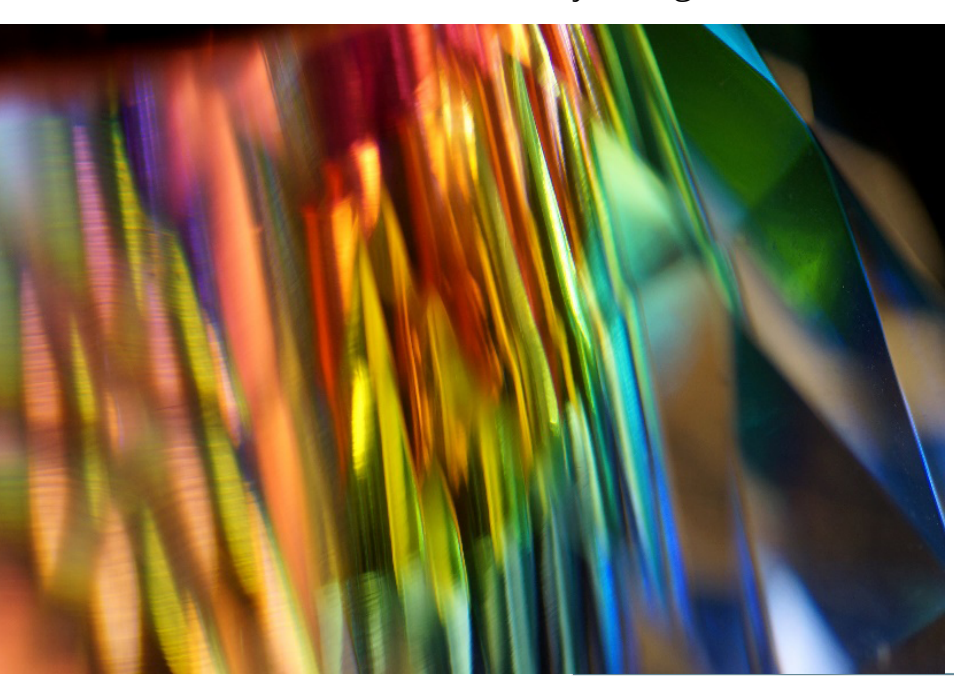
incorporadas en diversas expresiones culturales, hablan de la relevancia de la luz como fenómeno físico y a la vez como símbolo universal a lo largo del tiempo.

\section{lluminación}

Con referencia a este artículo, la iluminación se entiende como la aplicación de la luz para contribuir al desempeño visual de las personas en sus diversas actividades. Las asociaciones profesionales dedicadas a este campo de estudio se refieren a la iluminación como el arte y ciencia de la aplicación de la luz. 
Imagen 2. lluminación

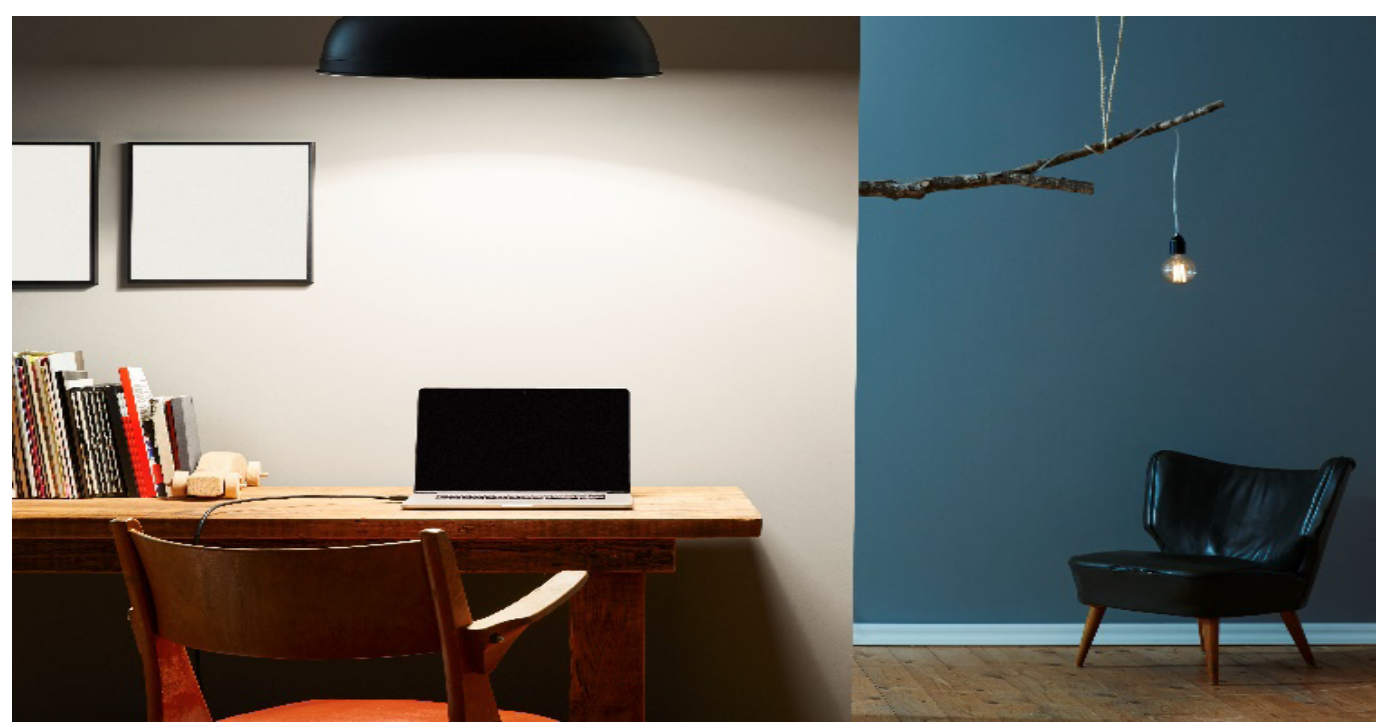

\section{Diseño de iluminación}

Es la especialidad dentro del campo del diseño cuyo objetivo consiste en desarrollar soluciones de iluminación que contribuyan a crear la experiencia visual de los espacios en beneficio de las personas.

El diseño de iluminación, particularmente el enfocado a integrar la luz artificial en los espacios arquitectónicos, urbanos y de paisaje, se ha desarrollado a partir de conocimientos técnicos provenientes de la ingeniería en iluminación aunados a una visión estética que tiene raíces en la iluminación teatral y escénica, así como en otras ramas del diseño.

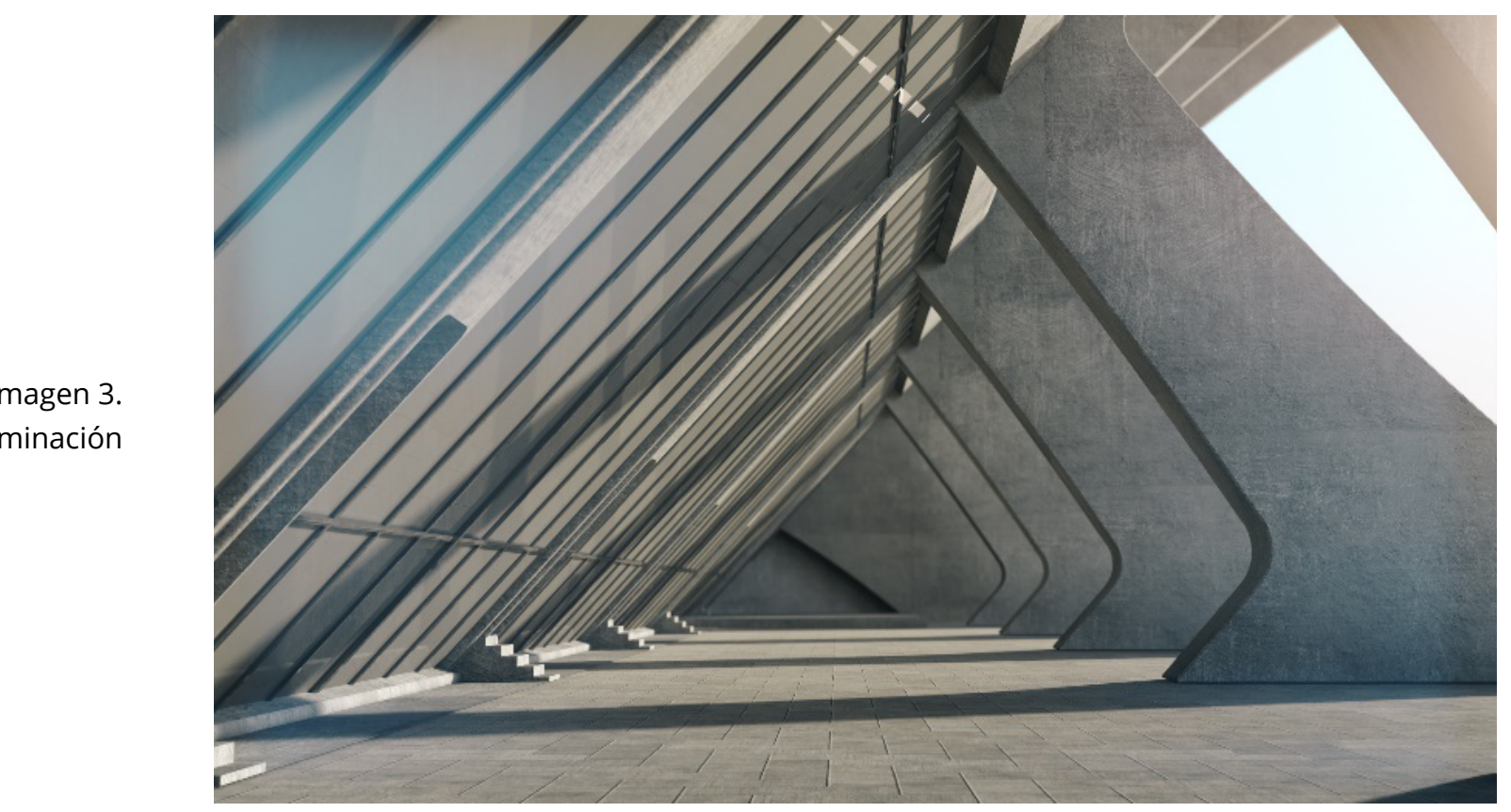

Imagen 3. Diseño de iluminación 


\section{Lámpara}

En la industria de la iluminación, una lámpara es una fuente luminosa. Las lámparas pueden ser de muy diversos tipos dependiendo del principio que utilizan para producir la luz. Por ejemplo, lámparas incandescentes son aquellas que generan energía luminosa bajo el principio de la incandescencia consistente en elevar la temperatura de un material hasta el punto en que emite luz. Otro caso son las lámparas fluorescentes que utilizan la capacidad del fósforo para transformar radiación ultravioleta en luz visible como sucede con los tubos de luz por fluorescencia. Coloquialmente, una lámpara sería llamada "foco" en México o "bombilla" en otros países de habla hispana.

Imagen 4.

Lámpara

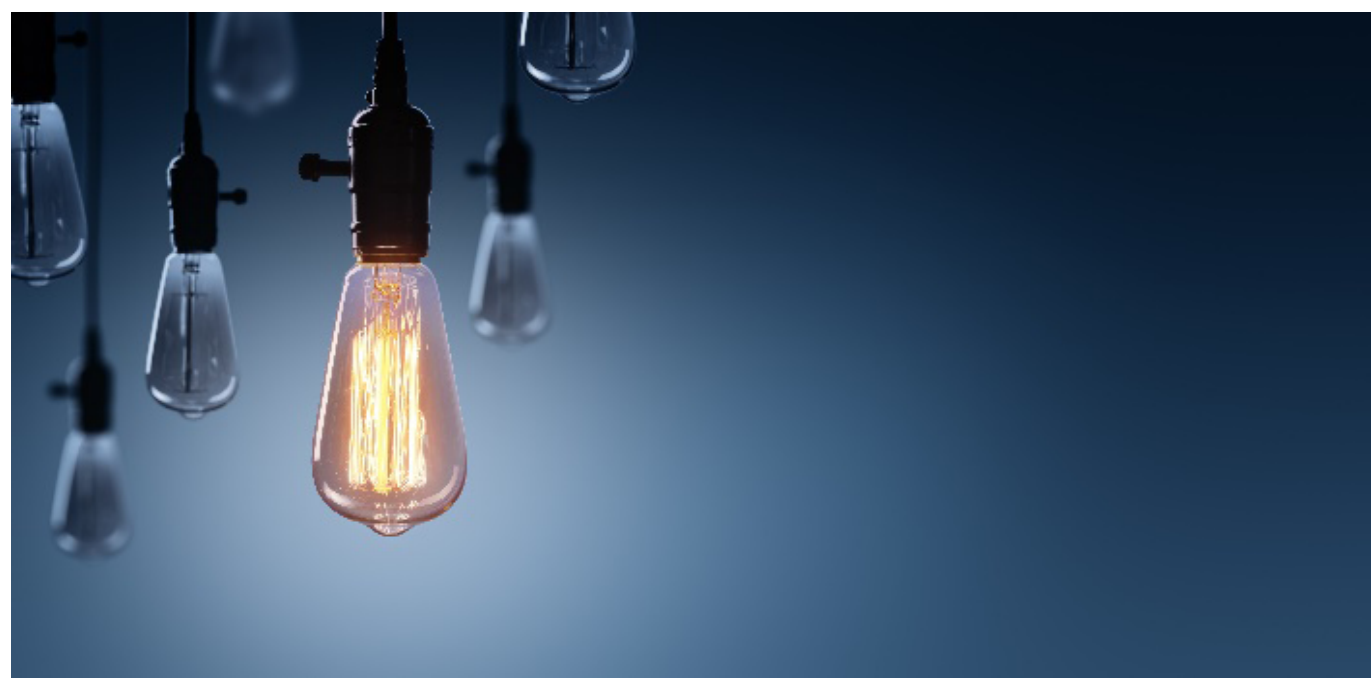

\section{Luminario}

Nuevamente, en la industria de la iluminación, un luminario es un equipo que integra una lámpara productora de luz, un sistema óptico como pueden ser reflectores o difusores, un cuerpo o carcaza y los componentes eléctricos necesarios para el funcionamiento de la fuente de luz. En el lenguaje común, el luminario es conocido como "lámpara" por ejemplo cuando nos referimos a un gabinete que contiene tubos fluorescentes utilizado en la iluminación de oficinas o bien, el equipo en la punta de un poste que sirve para el alumbrado público.

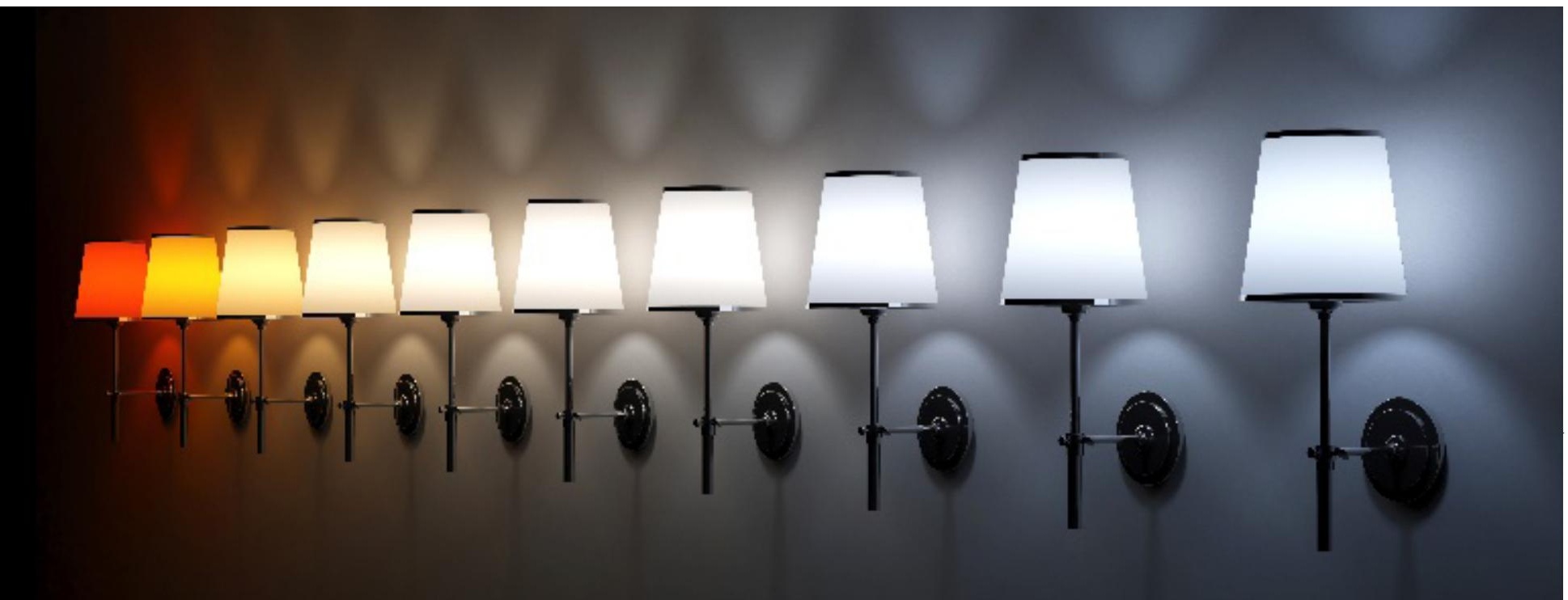


"La luz nos permite ver, nos estimula, informa y emociona. No existe la forma visual sin luz. Ella condiciona la forma en que percibimos el mundo y el cómo nos sentimos." (1)

Major et al., 2005: 1

\section{Un poco de historia}

El diseño de iluminación nace de forma casi espontánea como una especialización profesional. Entre sus antecedentes se encuentran la ingeniería en iluminación y el diseño de iluminación teatral, ambas profesiones resultado del uso práctico de la luz desde finales del siglo XIX.

El desarrollo de fuentes de luz eléctrica y su uso generalizado cambiaron la faz de las ciudades y la dinámica social preexistente. En 1906, Louis B. Marks impulsó la creación de la Sociedad de Ingeniería de lluminación de América del Norte (Illuminating Engineering Society of North America, IESNA) en la ciudad de Nueva York. Marks era un ingeniero eléctrico y uno de los primeros profesio-

Imagen 6.

La ciudad de Nueva York en 1951. nales que trabajaron como consultores en iluminación; en una carta dirigida a diversos colegas de la naciente industria, propuso la formación de la sociedad para "aquellos que estén especialmente interesados en las cuestiones de la luz y su distribución" (DiLaura, 2006: 10).

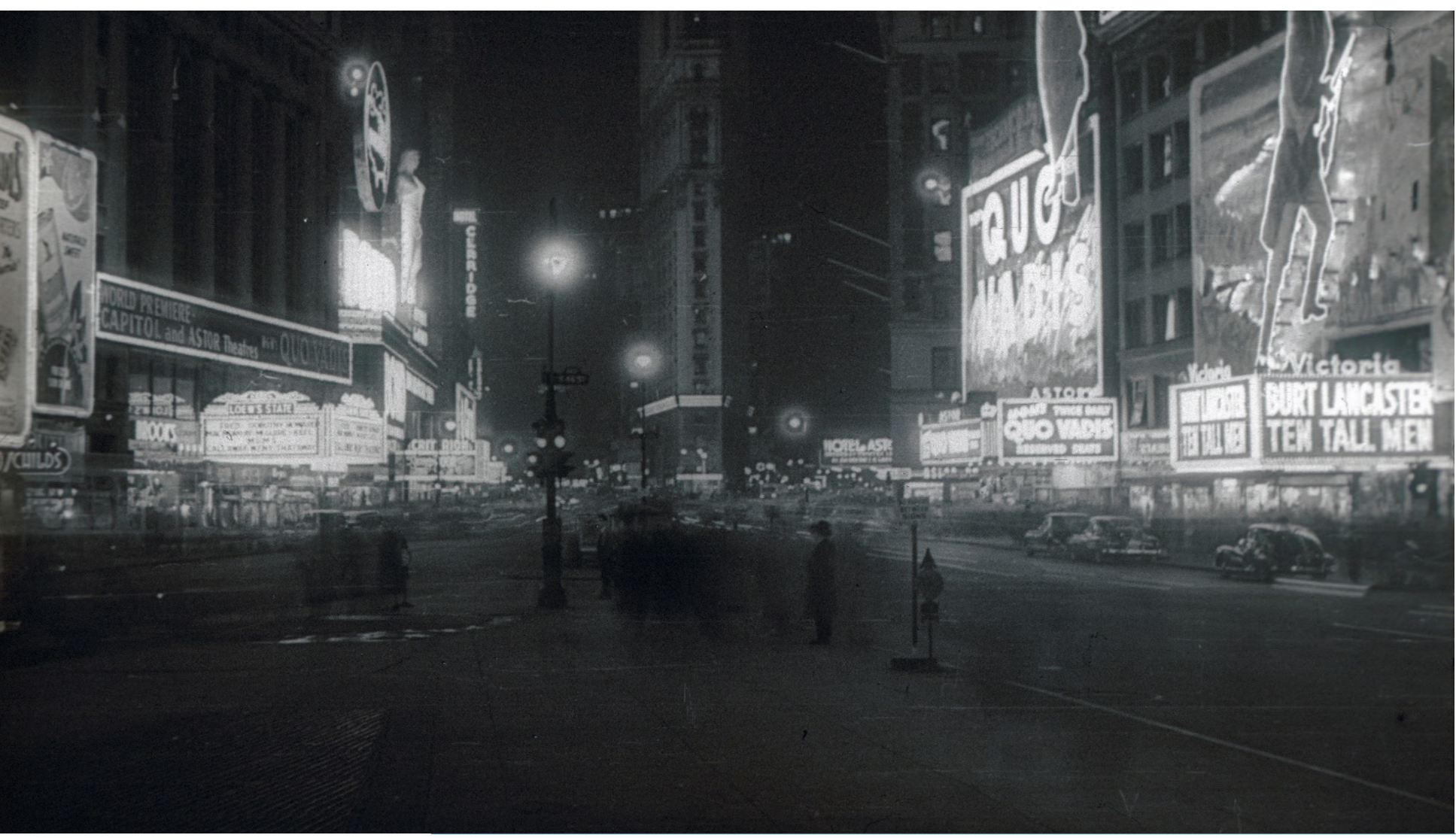


Imagen 7.

Richard Kelly
La ingeniería en iluminación nació y, a partir de ese momento, la IESNA se avocó al estudio de la luz y de su aplicación. De forma muy acertada, los fundadores decidieron crear comités especializados de expertos en diversos temas lumínicos para desarrollar conocimientos y difundirlos al público en general.

Al paso de los años, la IESNA se convirtió en la principal referencia técnica para los profesionales de la iluminación en Norteamérica y gran parte del mundo. Su manual (The Lighting Handbook) es conocido como la biblia de la iluminación y en su $10^{a}$ edición recopila gran parte de los conocimientos actuales sobre la física y óptica de la energía radiante, el diseño de la iluminación y las mejores prácticas de acuerdo con sus aplicaciones. En dicha edición se "ha tomado conocimiento de diversos asuntos que impactan el diseño hoy en día: límites en el uso de la energía, los efectos espectrales en la percepción y el desempeño visual y la necesidad de ser flexibles en el proceso de determinar la iluminación que considera la edad del observador, la reflectancia de la tarea visual y su importancia" (DiLaura et al., 2011: prefacio).

A partir de las aportaciones técnicas de la IESNA, diversos profesionales adoptaron la ingeniería en iluminación como su actividad principal y comenzaron a optimizar la aplicación de la luz, desde su generación por diversas fuentes lu-

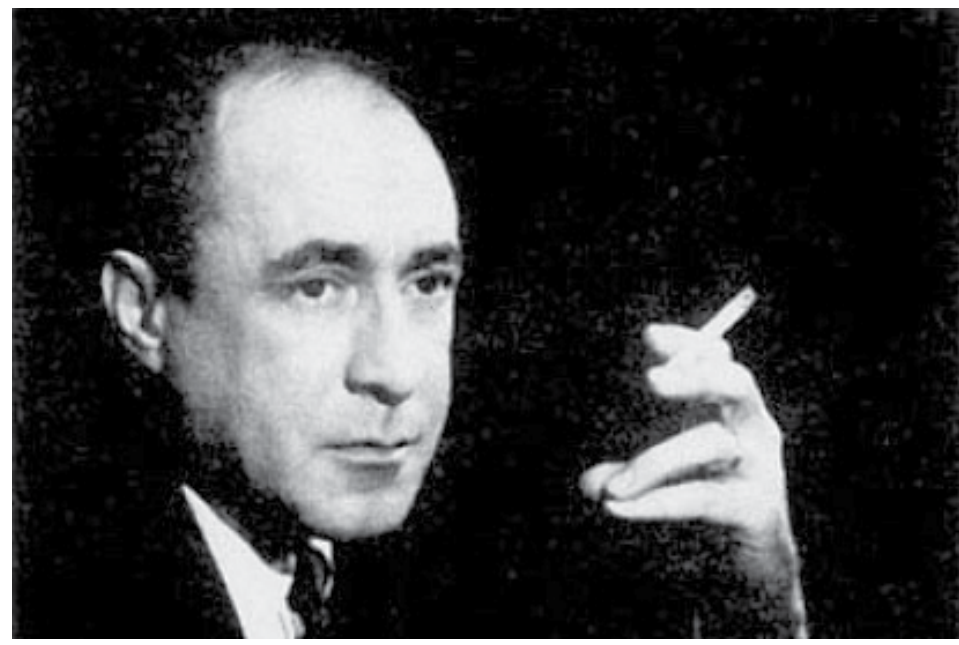
minosas hasta su uso en tareas de alto desempeño.

La relevancia de esta asociación consiste en su capacidad para desarrollar y divulgar conocimiento de manera abierta a toda la comunidad de la industria lumínica y de campos de estudio afines.

Pero no fue sino hasta la década de 1950 que un arquitecto comenzó de manera formal y consistente a aplicar los principios del diseño a la iluminación. Richard Kelly "entendió la capacidad de la luz para dar forma al espacio y crear una sensación de conciencia visual que pudiera evocar un rango de emociones humanas" (Donoff, 2006: 5).

\section{Creando las bases del diseño de iluminación}

En 1952, durante una reunión conjunta del Instituto Americano de Arquitectos (American Institute of Architects, AIA), la Sociedad de Diseñadores Industriales (Society of Industrial Designers, SID) y la Sociedad de Ingeniería en lluminación de Norteamérica ya mencionada previamente; Kelly compartió sus ideas en un ensayo titulado "Lighting as an Integral Part of Architecture" (La iluminación como una parte integral de la arquitectura) (1952: 24-26). 
Imagen 8.

Focal glow.

Fuente: $\underline{\text { ERCO. }}$

Imagen 9.

Ambient luminescence.

Fuente: ERCO.

Imagen 10.

Play of brilliants.

Fuente: $\underline{\text { ERCO}}$.
Los principios establecidos por Kelly con respecto al diseño de iluminación, incluidos en dicho ensayo, describen los tres componentes básicos de una escena visual, a saber:

- Focal glow. De difícil traducción, el término se refiere a una sensación de brillo que atrae nuestra atención como la luz que ilumina el escenario en un teatro, el baño de luz que recibimos cuando leemos bajo una lámpara en nuestro sillón favorito o la fogata en el campo que captura no sólo nuestra visión sino toda nuestra atención para socializar en torno a ella.

Kelly menciona en su ensayo que el focal glow une partes diversas, vende mercancía, crea jerarquía y ayuda a la gente a ver lo importante.

- Ambient luminescence. Es la luz del ambiente, la envolvente lumínica en nuestro campo visual es suave y uniforme, parece venir de las superficies propias y no de una fuente externa. Kelly se refiere a este factor como la luz ininterrumpida de una mañana nevada en el campo, la luz de la niebla en el mar y la bruma en un ancho río donde la rivera, el agua y el cielo se vuelven indistinguibles.

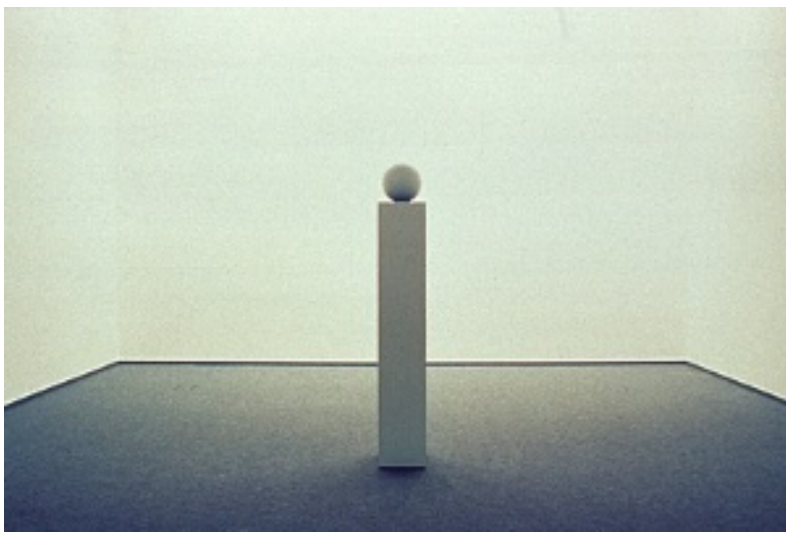

Para Kelly, la luz ambiental produce imágenes planas, sin sombras en las cuales la forma desaparece, pero a la vez evoca la libertad del espacio y sugiere infinidad. En las personas produce quietud y tranquilidad.

- Play of brilliants. El juego de la luz, de los puntos brillantes como las marquesinas de los viejos cines, los candiles de cristal en los salones de fiestas y la flama de una vela. También se trata del brillo del sol sobre el espejo de agua en una fuente o un vitral luminoso en una catedral.

Aquí, el autor habla de un efecto luminoso que estimula el nervio óptico y después de él el cuerpo y el

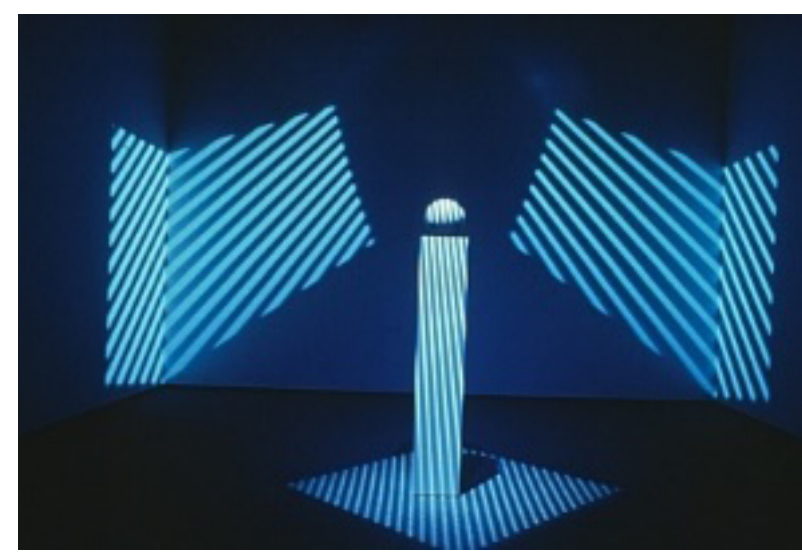


espíritu, haciendo alusión al efecto que la percepción de la luz tiene en nuestras emociones y su impacto en nuestras sensaciones.

La enunciación de estos tres principios marcó sin duda el punto de partida y la formalización de una nueva especialidad que hace uso de los recursos técnicos para generar emociones y sensaciones al integrar la luz en un espacio arquitectónico. Por primera vez, un arquitecto define principios que relacionan el valor de la iluminación con el espacio y las personas que lo habitan. Anteriormente, el enfoque predominante en la iluminación era exclusivamente el técnico que tiene por objetivo optimizar los recursos lumínicos en función del desempeño visual. La aportación de Richard Kelly desde el punto de vista teórico, pero también mediante los proyectos que realizó, marca un giro definitivo en la iluminación integrando la percepción visual, la relevancia del espacio y el impacto en las personas.

Y la obra de Kelly corresponde muy bien con el título de este artículo ya que fue arquitecto por educación profesional, diseñador de iluminación por elección de práctica profesional y educador por vocación, capaz de sintetizar ideas ob-

Imagen 11. Las luces de París tenidas en la experiencia para transformarlas en principios transmisibles a sus colegas y las generaciones posteriores.

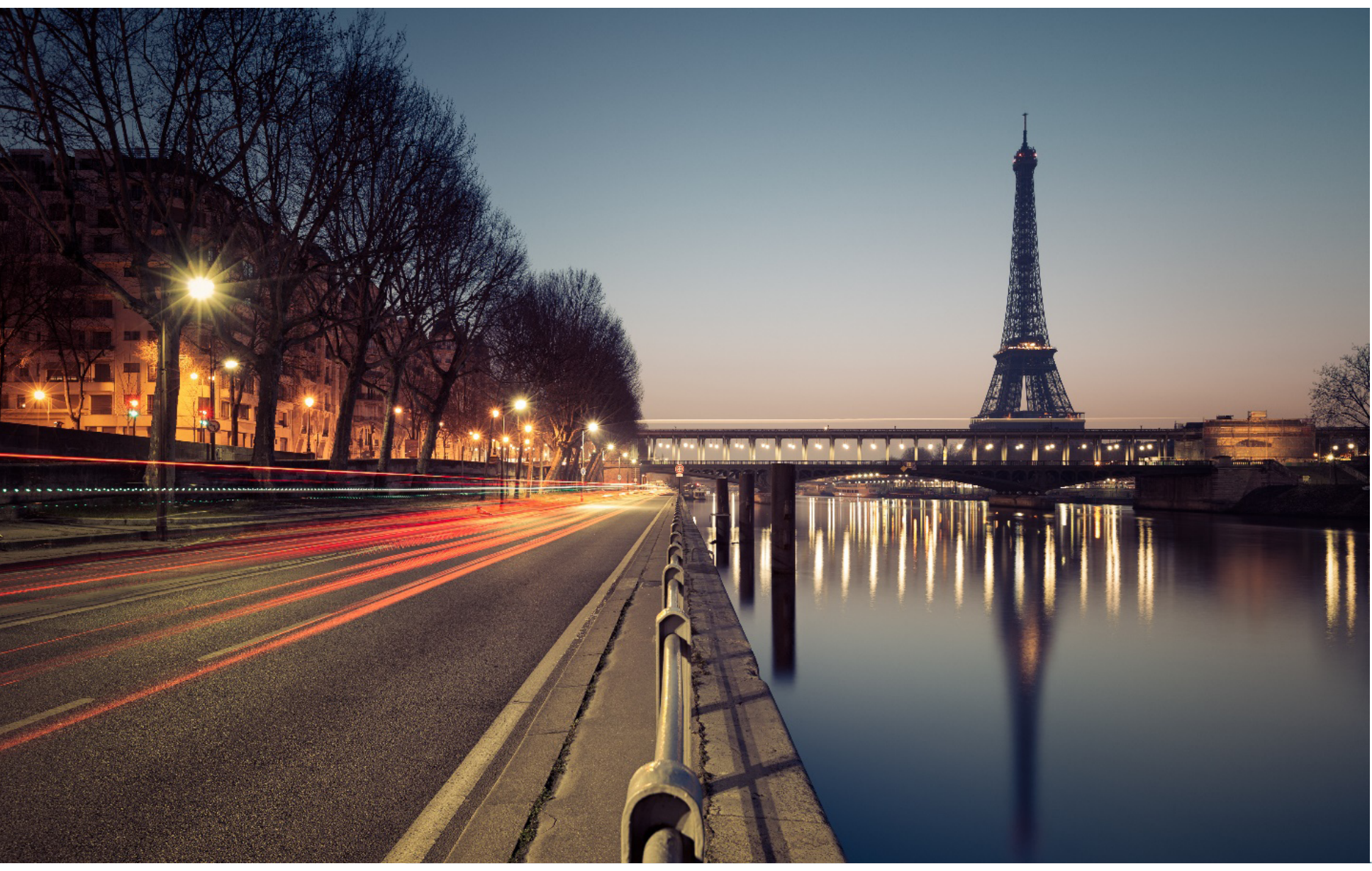


El caso de Kelly no es único. De una forma un tanto orgánica, la evolución del diseño de iluminación y la incorporación de los practicantes ha seguido una ruta similar, como lo comenta Francois Chaslin en su introducción al libro La Conception Lumière (El diseño de iluminación). Chaslin hace un recuento desde finales del siglo XIX y de la forma en que las farolas de aceite en las calles de París cambiaron a fuentes eléctricas. De manera casi poética, describe cómo la luz artificial cambió la vida de la sociedad, afirmando que hemos matado a la noche. Y se refiere a los diseñadores de iluminación diciendo: "la nueva profesión del diseño de iluminación apareció, de estos 'iluminadores', algunos habían sido 'iluministas' en el sentido técnico, otros ingenieros de diversas áreas, otros venían del cine o la fotografía, unos más eran artistas, decoradores, escenógrafos, arquitectos o diseñadores [...] a falta de espacios educativos de formación y de estatus profesional, se han agremiado y, después de algunos años, elaborado una cultura común" (Chaslin, 2017)

El relato de Chaslin corresponde a sus observaciones de las actividades de los especialistas de la iluminación en Francia, pero sin duda es representativo de lo que ha ocurrido en otros países, incluido México.

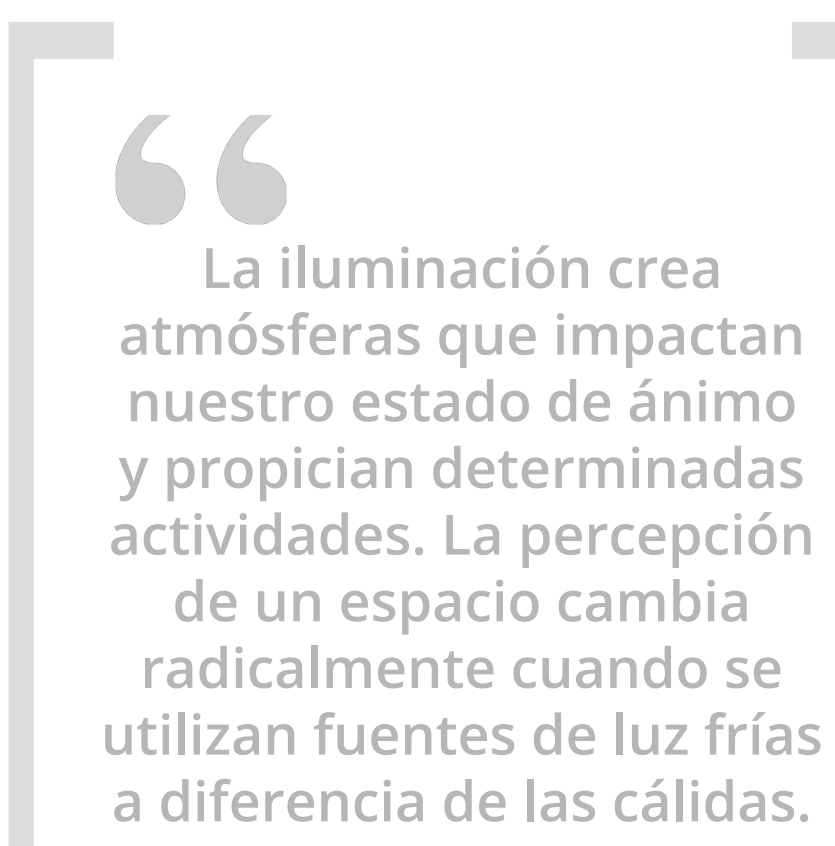

\section{Crecimiento profesional y educativo en México}

Los primeros diseñadores de iluminación en nuestro país comenzaron a hacer notar su presencia en la década de 1980, combinando profesionales provenientes de campos de la ingeniería, principalmente la eléctrica, con arquitectos, diseñadores industriales e inclusive profesionales de otros campos cuyo interés por la luz creció de manera notable.

Es de destacar por su liderazgo en esta materia al Arq. Gustavo Avilés, quien desde sus inicios como diseñador de iluminación logró atraer mucha atención sobre el potencial de la luz para transformar espacios, crear impacto visual y llevar la aplicación de la luz a una dimensión superior. 
El Diseño de lluminación como una especialidad con raíces en diversas disciplinas se desarrolla y evoluciona en la praxis para luego ser analizada, valorada y sistematizada por sus propios ejecutantes y convertida en disciplina académica.

Este proceso no es ajeno al de otras disciplinas, sin embargo, visto en el tiempo pareciera que actividades como la ingeniería, arquitectura, ciencias de la salud, ciencias sociales, etcétera, hubieran tenido su origen en la academia para, luego de haber instruido grupos de aspirantes, nutrir el campo profesional con nuevos practicantes.

¿Cómo se define una profesión?, ¿de qué manera se identifica una actividad humana como práctica profesional?

Evidentemente, aquellas especialidades que cuentan con una formación académica universitaria son reconocidas precisamente como profesiones y se acreditan mediante el título otorgado por la institución. En el mundo profesional es indispensable identificar el programa académico que se ha cursado, la institución que lo respalda y el título que se ha obtenido para poder afirmar que se es médico, historiador, diseñador industrial, administrador, etcétera.

Hablando del caso de México, durante la ya citada década de 1980, la Universidad Nacional Autónoma de México (UNAM) impartió algunos de los primeros cursos formales sobre la luz y sus aplicaciones a través de la Dirección de Educación Continua de la Facultad de Ingeniería. Los incipientes diseñadores de iluminación, entre los cuales se encuentra el autor de este artículo, asistieron con gran interés a estos cursos preparados y presentados por colegas que habían logrado sistematizar los conocimientos teóricos y prácticos de sus propias actividades para compartirlos en un ambiente académico.

Son de hacer notar las aportaciones del Ing. Sergio García Anaya como responsable de las materias de tecnología y fotometría, así como del Arq. Enrique Quintero a cargo del diseño de iluminación. Ambos iniciaron sus actividades profesionales en empresas especializadas, una fabricante de lámparas y la otra de luminarios respectivamente, tomando cursos impartidos de forma privada por las casas matrices en Estados Unidos y complementando con la información proveniente de la IESNA. No es casual que los tres profesionales mencionados sean en la actualidad los coordinadores de la especialidad en iluminación de la Facultad de Arquitectura de la UNAM.

Ejemplos similares se pueden encontrar en otros países de los cuales es notable el caso de David DiLaura, un ingeniero eléctrico especializado en iluminación que impartió cursos sobre la materia por más de 25 años en la Universidad de Colorado, creando un auténtico semillero de especialistas con impacto continental. Las aportaciones de DiLaura incluyen el desarrollo de los algoritmos empleados en los programas informáticos de cálculos de iluminación (sus alum- 
nos son en la actualidad los principales desarrolladores de estos programas), innumerables ponencias y artículos técnicos, además de ser el editor general de la $10^{a}$ edición del manual de la IES (en años recientes, el acrónimo se simplificó retirando las iniciales correspondientes a Norteamérica). Algunos mexicanos destacados en la industria como el Ing. Antonio Garza son egresados de la especialidad de la Universidad de Colorado y se han convertido posteriormente en profesores de materias de iluminación en múltiples instituciones académicas.

La teoría y práctica del diseño de iluminación, y por lo tanto los programas académicos desarrollados sobre la materia, incluyen conocimientos científicos proveniente de la física de la luz, desarrollos tecnológicos de la industria a nivel eléctrico y electrónico además de aportaciones de las ciencias médicas, particularmente en lo relativo a la fisiología de la visión humana que investiga los efectos visuales y no visuales de la luz. En la actualidad, las ciencias biológicas y ambientales aportan también datos relevantes a la aplicación de la luz y su impacto en los seres vivos y el medio ambiente.

Como disciplina creativa y de diseño, la transferencia de metodología y perspectiva propias de la Arquitectura y actividades afines como las artes escénicas, el diseño de interiores, el paisajismo, el Urbanismo e incluso el diseño de espacios comerciales o de exposición museográfica, conforman la visión de una especialidad que integra campos de conocimiento múltiples y se desarrolla como una actividad única.

\section{El reconocimiento de la profesión}

Los profesionistas y practicantes de una especialidad que no está acreditada por un programa académico establecido enfrentan retos para obtener el reconocimiento a sus capacidades y competencias. Los diseñadores de iluminación han entendido la necesidad de poner en valor esta especialidad y lo han logrado mediante diversas acciones.

En primer término, el diseño de iluminación ha conjuntado conocimientos de manera consistente y los ha sistematizado. Resultado de ello son los cursos de especialización ya mencionados, además de algunos muy relevantes a nivel internacional como los del Instituto Real de Tecnología de Suecia (KTH), la Universidad Politécnica de Madrid o la Universidad de Wismar, todos ellos en Europa y de los cuales hay un buen número de mexicanos egresados.

Caso especial es el de la Escuela de Diseño Parsons (Parsons School of Design) cuya maestría en Bellas Artes y Diseño de lluminación es cada año fuente principal de nuevos profesionistas para la industria de la iluminación; de sus filas han egresado importantes profesionales mexicanos, algunos de los cuales forman parte de destacadas firmas de diseño en México y el extranjero. 
Los practicantes del diseño de iluminación cuentan con asociaciones profesionales acreditadas que los agremian. De ellas cabe destacar la Asociación Internacional de Diseñadores de lluminación (International Association of Lighting Designers, IALD) cuya labor en favor del reconocimiento de la profesión es fundamental en la actualidad a nivel internacional. Por mencionar un ejemplo, la IALD ha logrado posicionarse como un consultor para la Comisión Europea, parte de los órganos de gobierno de la Unión Europea, en temas relacionados a la iluminación. Tradicionalmente, han sido los fabricantes quienes tienen peso en la toma de decisiones y definición de normas, pero hoy en día las asociaciones de profesionales elevan la voz de sus miembros buscando tener una influencia positiva en el ámbito social y político.

Cabe destacar que en nuestro país la sección México de la IES y la región México de la IALD son activas y promueven de manera continua la cultura de la iluminación, el mejoramiento de la práctica profesional y la difusión del conocimiento de esta especialidad. Ejemplos de ello son el Seminario IES que cada año se celebra en la Ciudad de México y que permite difundir conocimiento actualizado a nivel nacional e internacional sobre la luz y la iluminación. En este seminario, la región México de la IALD colabora desarrollando actividades que ponen a los estudiantes en contacto directo con especialistas técnicos y de diseño a nivel internacional. Otras actividades son los cursos de iluminación básica y avanzada, las mesas redondas en exposiciones de la industria eléctrica y de iluminación, así como las ponencias de miembros de ambas asociaciones en foros muy diversos. Para información sobre estas actividades se pueden consultar los siguientes sitios web: www.iesmexico.org y www.iald.org en la sección de regiones.

La influencia de la comunidad profesional mexicana ha sido relevante a nivel de Latinoamérica, Norteamérica e incluso en Europa por la constante participación y logros de connacionales en eventos, proyectos y colaboraciones gremiales. Inclusive, la promoción del diseño de iluminación a nivel de medios ha fomentado el desarrollo de un programa de reconocimientos a proyectos de iluminación iberoamericanos por parte de medios electrónicos. De igual forma, la participación de mexicanos ha sido constante y relevante en el evento Encuentro Iberoamericano de Lighting Design organizado por un grupo de profesionales voluntarios sin fines de lucro que inició en Valparaíso, Chile en 2010 y ha pasado por Querétaro, México; Medellín, Colombia y Ouro Preto, Brasil. El próximo Encuentro tendrá lugar a partir del 21 de marzo en la ciudad de Colonia de Sacramento, Uruguay con la participación de más de 200 profesionales de la iluminación de toda Iberoamérica. Más detalles se pueden consultar en el sitio web: www.eild.org.

Una forma adicional de lograr reconocimiento y validación para una profesión es el desarrollo de certificaciones. A este respecto, el programa de Certificación de Diseñadores de lluminación (Certified Lighting Designer, CLD) fue iniciado internacionalmente hace dos años y se encuentra en fase de crecimiento; hoy en día, hay 40 diseñadores reconocidos internacionalmente como CLD de los cuales dos se encuentran en México. 


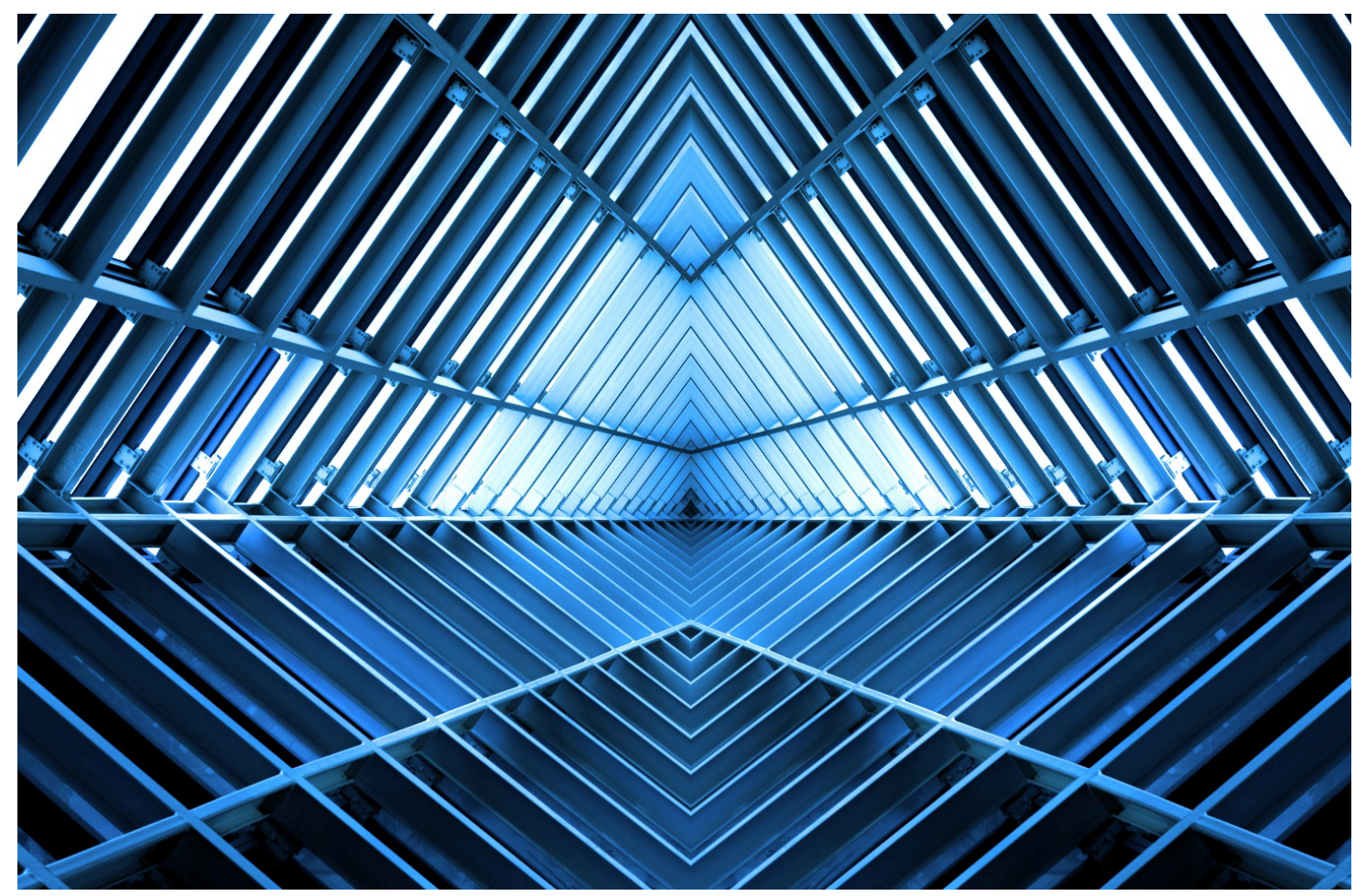

\section{Luz y sociedad}

El fin altruista de toda profesión está relacionado con su impacto en el ser humano, la sociedad y el medio ambiente. El diseño de iluminación tiene efectos en el desempeño visual de las personas, por ejemplo, en espacios de trabajo, pero también en actividades cotidianas en cualquier ámbito. Gracias a una correcta iluminación, las personas disfrutan sus alimentos sentados a la mesa de un restaurante, se desplazan de forma segura por las calles de una ciudad, aprecian una muestra de arte en una galería, conviven con sus amigos en la sala de estar de su casa bajo un ambiente cálido y acogedor, revisan con detalle las propiedades de la mercancía que compran en una tienda o admiran la belleza de un monumento histórico en el paisaje nocturno de su comunidad.

La iluminación crea atmósferas que impactan nuestro estado de ánimo y propician determinadas actividades. La percepción de un espacio cambia radicalmente cuando se utilizan fuentes de luz frías a diferencia de las cálidas. La luz uniforme nos comunica sensaciones distintas a la luz de acento. La dirección de la luz crea sensaciones, por ejemplo, cuando es ascendente crea sombras dramáticas y llama definitivamente nuestra atención. Las atmósferas son versiones distintas de un mismo espacio modificadas por la luz, la cual muestra su potencial transformador y su efecto en la vida del ser humano.

Finalmente, la luz tiene significados que interpretamos de manera intuitiva en ocasiones y consciente en otras. Mediante la iluminación, un espacio de culto 
adquiere carácter, invoca sensaciones y nos dispone a la introspección. La luz nos guía, atrae, captura y mueve, nos indica el camino y sin duda la utilizamos en nuestro favor. En el espacio público, la luz nocturna nos permite reunirnos y socializar, es la iluminación el factor que habilita la vida de la ciudad y sus habitantes cuando el sol se ha ocultado, y es la luz la que también nos relaciona con la oscuridad y nuestro reloj biológico, sin ella nuestro sistema nervioso no podría ponernos en alerta, disponernos a la actividad ni bajara el ritmo para enviarnos a descansar cuando el sol se oculta.

Citando a Mark Major y Jonathan Speirs de su libro Made of Light, "Para todo aquello que hacemos en la vida, la luz tiene un papel. La luz es un instrumento de comunicación; es energía y es magia. La luz es vida. Nuestro mundo está hecho de Luz" (2005: 1).

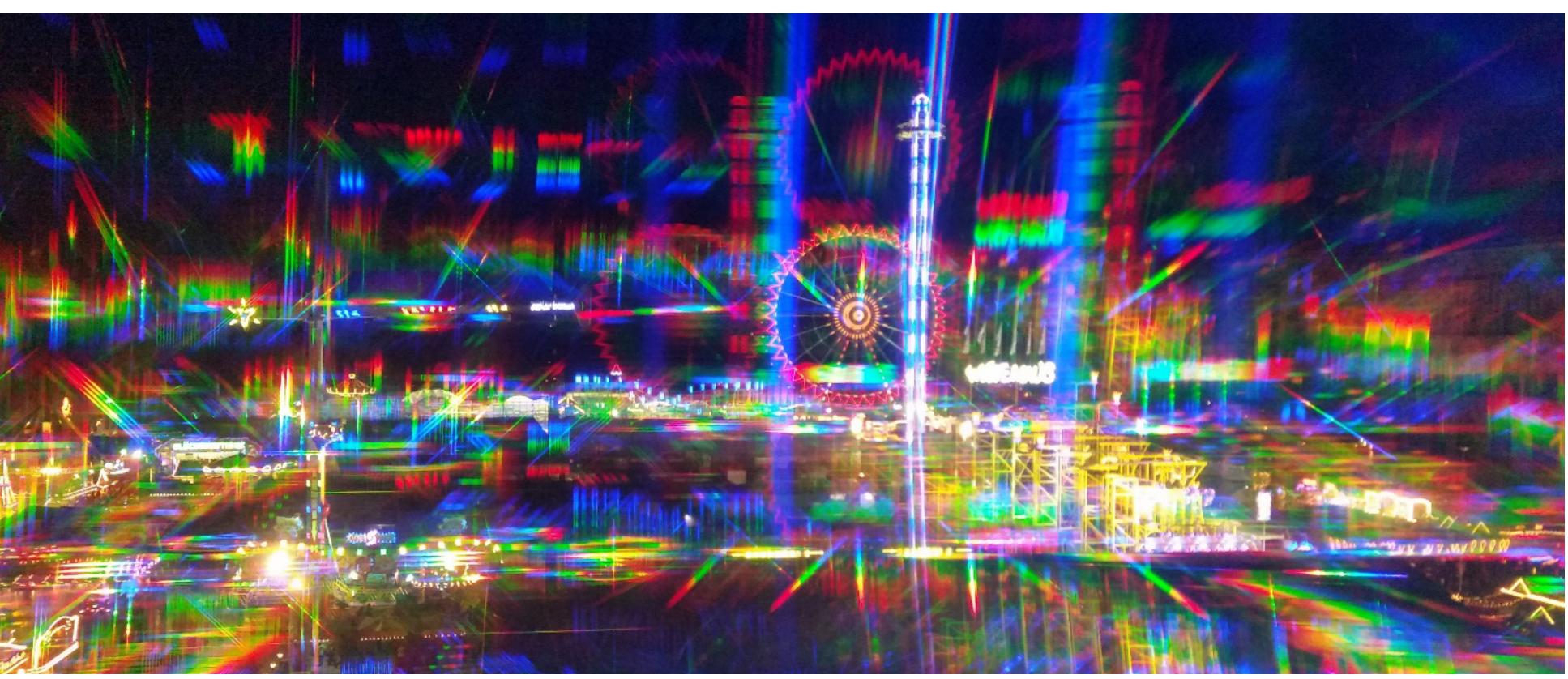

\section{Referencias}

* CLD Commission (2015). Manual del Candidato. Para diseñadores de iluminación arquitectónica en ejercicio. Chicago: Certified Lighting Designer, CLD. Recuperado de http://cld.global/CLD/media/media/CLD-Handbook-Edits-June-17 ES-ES.pdf.

- DiLaura, D. (2006). A History of Light and Lighting. EE. UU.: Illuminating Engineering Society.

- DiLaura, D., Houser, K., Mistrick, R. y Steffy, G. (2011). The Lighting Handbook. 10th Edition. Nueva York, EE. UU.: Illuminating Engineering Society. 
- Donoff, E. (2006). Richard Kelly's Three Tenets of Lighting Design. Architectural Lighting, AL 30th Anniversary.

- James, P. (2018). Editorial. Mondo ARC. ILDS, 18.

* Kelly, R. (1952). College Art Journal. EE. UU.: College Art Association.

* LIB Chaslin, F. (2017). La Conception Lumière. París, Francia: Moniteur.

* Major, M., Speirs, J. y Tischhauser, A. (2005). Made of Light. Basel, Switzerland: Birkhauser.

\section{Cómo citar este artículo}

* Palacio, Víctor (2018). Diseño de iluminación: desarrollo, práctica y educación. Revista Digital Universitaria (RDU). Vol. 19, núm. 3 mayo-junio. DOl: http://doi. org/10.22201/codeic.16076079e.2018.v19n3.a2. 